\title{
Qualidade de vida de idosos com incontinência urinária
}

\author{
Quality of life in senior citizens with urinary incontinence
}

\author{
Karin Viegas ${ }^{1}$, Márcia Welfer², Gabriela Denes Lucho ${ }^{3}$, Cibele Cardenaz de Souza ${ }^{4}$, \\ Beatriz Regina Lara dos Santos ${ }^{5}$, Denizar Alberto da Silva Melo ${ }^{6}$, Mara Regina Knorst ${ }^{7}$, \\ Thais de Lima Resende ${ }^{8}$, Marion Creutzberg ${ }^{9}$
}

\begin{abstract}
RESUMO
Objetivo: Verificar a prevalência de incontinência urinária (IU) em amostra de idosos de Porto Alegre/RS, relacionando a qualidade de vida entre incontinentes e continentes.

Materiais e Métodos: Estudo transversal com 509 idosos. Foram utilizados o Index de Barthel e uma questão fechada para verificação da IU e o WHOQOL-Bref. Os dados foram analisados pelo SPSS 11.5. Estudo desenvolvido com base na Resolução 196/96-CNS/MS.

Resultados: Por meio do Index de Barthel, $24 \%$ apresentam IU; dentre as mulheres, $29,4 \%$ e $10,3 \%$ dos homens. Na questão única, $50 \%$ referiram IU. Houve relação entre sexo e incontinência, com prevalência maior entre as mulheres $(p<0,001)$ e entre IU e avaliação global da QV e satisfação com a saúde $(p<0,05)$. Os resultados confirmam achados de outros estudos.
\end{abstract}

Conclusão: $O$ planejamento e investimento em intervenções terapêuticas, com abordagens interdisciplinares, são imprescindíveis para melhoria da QV dessa população.

Palavras-chave: idoso; envelhecimento; incontinência urinária; qualidade de vida.

\section{ABSTRACT}

Objective: To assess the prevalence of urinary incontinence (UI) in an elderly citizen sample in Porto Alegre, RS, as well as to determine whether there is a difference in the quality of life between incontinent and continent people.

Materials and Methods: A crosscut survey with 509 elderly citizens. Barthel's Index and a closed question were used to evaluate the UI and WHOQOL-Bref. Data were analyzed by SPSS 11.5. The study was carried out according to Resolution 196/96-CNS/MS.

Results: Analyzing the Barthel's Index, 24\% presented UI; among women, $29.4 \%$ and $10.3 \%$ among men. In the single question, $50 \%$ have mentioned UI. There was an association between sex and incontinence, prevailing the women $(p<0.001)$, and between $\mathrm{UI}$ and the quality of life global assessment and health $(p<0.05)$. The results confirm the findings of other surveys.

Conclusion: The quality of life and health are better among the continent subjects. Planning and investment in therapeutic interventions, with interdisciplinary approaches, are essential to improve the quality of life in such population group.

Keywords: aged; aging; urinary incontinence; quality of life.

${ }^{1}$ Enfermeira. Doutora em Gerontologia Biomédica. Professora da Faculdade de Enfermagem, Nutrição e Fisioterapia (FAENFI) da PUCRS.

${ }^{2}$ Enfermeira. Mestre em Gerontologia Biomédica. Professora da Factum. Bolsista AT-CNPq.

${ }^{3}$ Fisioterapeuta. Mestranda em Gerontologia Biomédica. Bolsista AT-CNPq.

${ }^{4}$ Fisioterapeuta. Graduado em Fisioterapia pela PUCRS.

${ }^{5}$ Enfermeira. Doutora em Educação. Professora Adjunta da FAENFI/PUCRS.

${ }^{6}$ Fisioterapeuta. Doutor em Ciências da Saúde. Professor Adjunto e Coordenador do Curso de Fisioterapia da PUCRS.

${ }^{7}$ Fisioterapeuta. Doutora em Gerontologia Biomédica. Professora Adjunta da FAENFI/PUCRS. Coordenadora do Centro de Reabilitação-PUCRS.

${ }^{8}$ Fisioterapeuta. Doutora em Ciências da Saúde. Professora Adjunta da FAENFI/PUCRS e UNISINOS.

${ }^{9}$ Enfermeira. Doutora em Gerontologia Biomédica. Professora Adjunta e Vice-Diretora da FAENFI/PUCRS. 


\section{INTRODUÇÃO}

As doenças podem induzir com maior intensidade as modificações do envelhecimento que consequentemente se manifestam clinicamente, exteriorizando-se de maneira a tornar possível sua caracterização ${ }^{1}$. Dentre tais modificações a incontinência urinária (IU) é um problema de saúde significante para os idosos ${ }^{2}$. A força de contração da musculatura detrusora, a capacidade vesical e a habilidade de adiar a micção aparentemente diminuem no homem e na mulher. Contrações involuntárias da musculatura vesical e o volume residual pós-miccional aumentam com a idade em ambos os sexos. Entretanto, a pressão máxima de fechamento uretral, o comprimento uretral $e$ as células da musculatura estriada alteram-se predominantemente nas mulheres $^{3,4}$.

Além das alterações decorrentes do processo do envelhecimento, doenças próprias do indivíduo idoso também contribuem para o desenvolvimento de incontinência urinária, como por exemplo, a hiperplasia prostática benigna, que está presente em aproximadamente $50 \%$ dos homens com mais de 50 anos de idade, o diabetes e a insuficiência cardíaca estão entre os fatores que podem ser responsáveis pela $\mathrm{IU}^{5,6}$.

Embora a IU não coloque diretamente a vida das pessoas em risco, é uma condição que pode trazer sérias implicações de saúde, bem como, sociais, psicológicas e econômicas, afetando adversamente a qualidade de vida $(Q V)^{7}$. Estudos europeus ${ }^{8}$, norteamericanos $^{9}$ e brasileiros $^{10}$ demonstram importante prejuízo na QV de mulheres com incontinência urinária em diferentes faixas etárias. Em um aspecto geral, as mulheres com IU referem limitações físicas (praticar esporte, carregar objetos), sociais, ocupacionais e domésticas, influenciando negativamente no estado emocional e na vida sexual ${ }^{11,12}$. O desconforto social e higiênico dá-se principalmente pelo medo da perda urinária, o cheiro de urina, a necessidade de utilizar protetores (absorventes) e as trocas mais frequentes de roupas $^{11,12}$. A presença de sintomas irritativos, como a noctúria e a urgência, muitas vezes, atrapalham o sono e o relacionamento social, podendo ocasionar cansaço, depressão e isolamento social ${ }^{13}$.

Devido à relevância deste tema os objetivos deste trabalho são: a) determinar a prevalência de incontinência urinária (IU) em amostra de idosos de Porto Alegre/RS; b) analisar se os idosos com IU têm qualidade de vida diferente dos idosos não incontinentes; c) verificar se os idosos selecionados inicialmente para o estudo apresentam características demográficas, socioeconômicas e de saúde diferente entre os que compareceram para a segunda fase do estudo.

\section{MATERIAIS E MÉTODOS}

Esta pesquisa é um desdobramento de um inquérito populacional realizado nos idosos do município de Porto Alegre, que foi seguido por uma avaliação multiprofissional das condições de saúde dessa população. Esse estudo (EMIPOA - Estudo Multidimensional dos Idosos de Porto Alegre $)^{14}$ foi realizado pelo Instituto de Geriatria e Gerontologia (IGG) da Pontifícia Universidade Católica do Rio Grande do Sul (PUCRS), no período de dezembro de 2005 a setembro de 2006. A população-alvo foi constituída por indivíduos com idade igual ou superior a 60 anos, e a amostra foi baseada na estimativa da população para 2005. A sistemática da amostragem foi estratificada e proporcional por região censitária, com seleção aleatória do primeiro domicílio de cada região e sistematizada dos demais, sendo sorteado um idoso por domicílio.

Foram incluídos 1078 idosos na primeira fase do estudo, na qual foi realizado uma entrevista domiciliar por profissionais treinados do Serviço Social da PUCRS. Para isso, foi utilizado um questionário objetivo contendo informações demográficas gerais, sociais, culturais e econômicas, além de dados de saúde e atividades de vida diária. Todos os entrevistados foram convidados a participar da segunda fase do estudo, na qual foi realizada uma avaliação multidisciplinar por profissionais das faculdades de medicina, fisioterapia, farmácia, enfermagem, psicologia, educação física e nutrição. Nessa etapa, foram avaliados 509 idosos que aceitaram participar e compareceram para a coleta de dados na PUCRS, sendo excluídos aqueles com doença 
psiquiátrica ou déficit cognitivo severo ou com incapacidade de deambulação.

Todos os idosos foram questionados sobre ter a presença ou não de perda urinária. Para os participantes selecionados foram aplicados os instrumentos Index de Barthel ${ }^{15}$ e o WHOQOL-Bref ${ }^{16}$ para avaliar a grau de dependência e a qualidade de vida.

Com o Index de Barthel, modificado por Granger ${ }^{17}$, é uma medida genérica que avalia a capacidade funcional do indivíduo para executar atividades de vida diárias e atividades instrumentais e foi utilizado para avaliar o grau de incapacidade funcional dos indivíduos. Uma incapacidade orgânica não é condição suficiente para determinar dependência ${ }^{18,19}$. Dos 15 itens do Index de Barthel, foram acrescentados dois itens referentes à incontinência urinária e intestinal, não modificando a pontuação final. Tal inclusão esclarece o nível de dependência quanto aos cuidados necessários referentes ao controle urinário e intestinal.

$\mathrm{O}$ instrumento foi aplicado diretamente ao idoso, sem interferência do acompanhante. Todas as questões assinalam diferentes pontuações e ponderações, sendo que utilizamos a interpretação sugerida por Shah et al. ${ }^{18}$ : 100 é a pontuação máxima e indica independência; 91-99, dependência leve; 61-90, dependência moderada; 21-60, dependência severa; e contagens inferiores a 20 pontos, dependência total $^{17,20,21}$. Os pacientes com escore 100 são considerados independentes, não indicando que estão aptos a viverem sozinhos, mas são aptos a realizarem seus cuidados pessoais sem auxílio ${ }^{21}$.

Para medir a qualidade de vida dos idosos, foi utilizado o WHOQOL-Bref ${ }^{22}$, que consta de 26 questões, sendo duas questões gerais de qualidade de vida e 24 questões que representam um dos quatro domínios que compõem o instrumento original. Todas as questões apresentam cinco opções ordenadas, compondo um valor que varia de 1 a 5 , para cada resposta. A primeira questão reflete como o entrevistado avalia sua qualidade de vida, em uma escala com opções que vão de "muito ruim" a "muito boa". A segunda questão mede a satisfação do indivíduo com seu estado de saúde e tem escala que varia de "muito satisfeito" até "muito insatisfeito". As questões de 3 a 9 referem-se à influência da presença de dor, tratamento médico, aproveitamento da vida, sentido da própria vida, concentração, segurança e ambiente físico na qualidade de vida. Todas as respostas medem o quanto a pessoa tem sentido algo e varia de "nada" a "extremamente". As perguntas de 10 a 14 estão relacionadas ao quanto a pessoa tem se sentido ou é capaz de fazer alguma coisa (questionam energia para o dia-a-dia, aparência física, dinheiro, informações disponíveis e lazer). As opções de respostas vão de "nada" a "completamente". A pergunta 15 refere-se à capacidade de locomoção, cuja resposta varia de "muito ruim" a "muito bom". As perguntas de 16 a 25 medem a satisfação com vários aspectos da vida (sono, desempenho nas atividades diárias, trabalho, relações pessoais, vida sexual, apoio social, acesso a serviços de saúde, lugar que habita e meio de transporte), e sua resposta varia de "muito insatisfeito" a "muito satisfeito". A última pergunta referese à frequência com que apresenta sentimentos negativos e conta com uma escala que vai de "nunca" a "sempre". Cada aspecto representa uma das facetas do original WHOQOL-100.

Para o preenchimento do WHOQOL-Bref, foi solicitado que o idoso tivesse em mente seus valores, aspirações, prazeres e preocupações, tomando como referência as duas últimas semanas.

Os instrumentos foram aplicados por meio de entrevista individual, sendo pausadamente lida cada questão. Todos os entrevistados foram informados sobre o objetivo da aplicação do instrumento e do modo de aplicação.

Os dados coletados foram digitados e organizados em banco de dados especificamente desenvolvidos para o projeto, com conferência e correção posteriores da digitação, e analisados no software estatístico SPSS, versão 11.5 , sendo considerados significativos valores de $P$ iguais ou inferiores a $5 \%$. A prevalência foi calculada a partir da amostra, com respectivo intervalo de confiança de $95 \%$. Os resultados estão apresentados sob forma de média e desvio padrão da média para as variáveis contínuas e percentuais para as variáveis categóricas. Para verificar a relação entre as variáveis utilizou-se o teste de qui-quadrado. A correlação entre 
incontinência urinária e o Index de Barthel foi calculada pela correlação de Rho de Spearman. Para verificar a relação entre continência, incontinência, avaliação global da qualidade de vida e satisfação geral com a saúde foi utilizado o Teste $T$.

O estudo "Avaliação Transdisciplinar dos idosos de Porto Alegre", projeto conjunto entre Instituto de Geriatria e Gerontologia, seis Faculdades da PUCRS e Hospital São Lucas da PUCRS, foi aprovado pelo Comitê de Ética em Pesquisa da PUCRS, com o protocolo $n^{\circ}$ 031/2006-CEP. Os idosos participantes assinaram um Termo de Consentimento Livre e Esclarecido e a pesquisa foi conduzida dentro das normas da Resolução 196/1996 do Conselho Nacional de Ética em Pesquisa ${ }^{23}$.

\section{RESULTADOS}

Da amostra aleatória inicial de 1078 idosos, 479 completaram a investigação, sendo $343(71,6 \%)$ do gênero feminino. A média de idade foi de 70,3 anos \pm 7,8 anos.

Quando comparado com o grupo de idosos que não compareceu para avaliação, a distribuição por sexo foi semelhante e foi observado que a proporção de idosos com mais de 75 anos foi discretamente menor no grupo avaliado. A população estudada é em sua maioria branca, aproximadamente $2 / 3$ não completaram o $1^{\circ}$ grau e recebem até três salários mínimos, sendo estas características semelhantes nos dois grupos. Menos de $10 \%$ da população não tem renda própria, sendo esta proporção levemente maior no grupo que não foi investigado $(p<0,001)$. A autopercepção de saúde e a frequência das doenças e condições de saúde, questionadas no inquérito populacional, não mostraram diferenças estatisticamente significativas entre os idosos que realizaram e os que não realizaram a avaliação clínica na segunda fase do estudo. Entretanto, verificamos $43,2 \% \quad(p=0,006) \quad$ informaram urgência miccional no inquérito domiciliar (Tabela 1 ).

Ao serem perguntados acerca da presença de incontinência urinária, por meio do Index de Barthel ( $n=479), 364$ (71,5\%) idosos não apresentam episódios de incontinência urinária e 115 (24\%) idosos responderam que tem perda de urina, com nível de
TABELA 1. Comparação das

características demográficas, socioeconômicas e autopercepção de saúde entre os idosos na amostra aleatória de 1078 idosos do município de Porto Alegre - Brasil - 2006.

\begin{tabular}{|c|c|c|c|}
\hline VARIÁVEL & $\mathrm{N}(\%)$ & Avaliados & $P$ \\
\hline \multicolumn{4}{|l|}{ Sexo } \\
\hline Masculino & $304(28,2)$ & $136(28,4)$ & $0,243^{*}$ \\
\hline Feminino & $774(71,8)$ & $343(71,6)$ & \\
\hline \multicolumn{4}{|l|}{ Faixa etária } \\
\hline $60-64$ & $254(23,6)$ & $120(25,0)$ & $<0,001^{\$}$ \\
\hline $65-69$ & $203(18,8)$ & $117(22,9)$ & \\
\hline $70-74$ & $225(20,9)$ & $105(21,9)$ & \\
\hline $75-79$ & $196(18,2)$ & $79(16,5)$ & \\
\hline $80-84$ & $134(12,4)$ & $39(8,1)$ & \\
\hline 85 ou + & $66(6,1)$ & $19(3,9)$ & \\
\hline \multicolumn{4}{|l|}{ Raça } \\
\hline Branca/amarela & $893(82,8)$ & $381(79,5)$ & $0,391^{*}$ \\
\hline Parda/negra & $185(17,2)$ & $98(20,5)$ & \\
\hline \multicolumn{4}{|l|}{ Estado civil } \\
\hline Solteiro & $166(15,4)$ & $72(15,0)$ & $0,230^{*}$ \\
\hline Casado & $395(36,5)$ & $174(36,3)$ & \\
\hline Viúvo & $423(39,3)$ & $194(40,5)$ & \\
\hline Separado/ & $95(8,8)$ & $39(8,2)$ & \\
\hline Desquitado & & & \\
\hline \multicolumn{4}{|l|}{ Escolaridade } \\
\hline Nunca estudou & $123(11,4)$ & $46(9,6)$ & $0,364^{\$}$ \\
\hline $1^{\circ} \mathrm{grau}$ incompleto & $595(55,2)$ & $259(54,1)$ & \\
\hline $1^{\circ}$ grau & $142(13,3)$ & $63(13,2)$ & \\
\hline $2^{\circ} \mathrm{grau}$ & $126(11,7)$ & $66(13,7)$ & \\
\hline Superior & $91(8,4)$ & $45(9,4)$ & \\
\hline \multicolumn{4}{|l|}{ Renda própria } \\
\hline Sim & $958(88,9)$ & $454(94,9)$ & $<0,001^{*}$ \\
\hline Não & $61(5,6)$ & $11(2,3)$ & \\
\hline \multicolumn{4}{|l|}{$\begin{array}{l}\text { Auto percepção de } \\
\text { saúde }\end{array}$} \\
\hline Ótima & $143(13,3)$ & $59(12,2)$ & $0,779^{\$}$ \\
\hline Boa & $367(34,0)$ & $175(36,5)$ & \\
\hline Regular & $466(43,2)$ & $201(41,9)$ & \\
\hline Má & $45(4,2)$ & $20(4,1)$ & \\
\hline Péssima & $57(5,3)$ & $24(5,2)$ & \\
\hline \multicolumn{4}{|l|}{ Condições de saúde } \\
\hline $\begin{array}{l}\text { Perda urinária ao } \\
\text { esforço }\end{array}$ & $127(35,0)$ & $125(26,2)$ & $0,074^{*}$ \\
\hline Urgência miccional & $155(43,2)$ & $131(27,4)$ & $0,006^{*}$ \\
\hline
\end{tabular}

significância de $p<0,05$. Dos que têm IU, $100(20,9 \%)$ referiram perda ocasional com uma perda em $24 \mathrm{~h}$ e 15 $(3,1 \%)$ referiram perder mais que uma vez em $24 \mathrm{~h}$.

Ao avaliar a relação entre sexo e perda de urina, por meio do meio do Index de Barthel, verificou-se que quase três vezes mais mulheres apresentam IU do que os homens ( $29,4 \%$ das mulheres e $10,3 \%$ dos homens), apresentando-se estatisticamente significante, com $p<0,001$. A relação entre sexo e incontinência igualmente foi significativa nos resultados referentes à questão única, $(p<0,001)$, demonstrando prevalência maior de IU nas mulheres (Tabela 2). 
Dos que se dizem incontinentes 93,1\% realizam sozinhos os cuidados relativos à higiene e $6,9 \%$ necessitam de auxílio para execução desse cuidado. $O$ Index de Barthel, quanto à presença de incontinência classifica a pessoa como dependente. No entanto, incluiu-se a questão acerca das providências no sentido de que se observe que, mesmo que o resultado tenha um impacto sobre o escore que define o nível de dependência, essas pessoas não são dependentes, pois mantém a capacidade para o autocuidado. Observou-se que existe forte correlação entre o escore total do Index de Barthel e a presença de incontinência $(r=0,734$ e $\mathrm{p}<0,001)$.

Dos idosos que responderem ao Index de Barthel, 316 realizaram avaliação fisioterápica respondendo à questão sobre perda de urina. Destes, 158 (50\%) referiram ser incontinentes, sendo que $37 \%$ haviam referido continência no Index de Barthel. Enquanto, $7,5 \%$ dos idosos que haviam referido incontinência no Index de Barthel negaram diante da questão aberta. A contradição a respeito da IU verificada por meio do Index de Barthel e pela pergunta direta foi significativa $(p<0,001)$. Os dados coincidentes quanto à incontinência demonstram que 21,6\%, desses idosos, são incontinentes.

Quando verificada a associação entre a avaliação global da qualidade de vida e a incontinência urinária pelo Index de Barthel e pela questão única, observou-se que houve diferença significativa entre idosos com e sem incontinência, na qualidade de vida. Os idosos com incontinência referem qualidade inferior aos idosos sem incontinência, com $p=0,002$ no Index de Barthel e $p=0,021$, na questão única.

Foi observado que a média da QV geral, que analisa as duas primeiras questões do instrumento, referente a autoavaliação da qualidade de vida e autoavaliação da saúde, quando comparado com o Índex de Barthel, foi maior nos idosos sem incontinência urinária $(p<0,001)$.

\section{DISCUSSÃO}

É importante destacar algumas limitações do estudo que podem interferir nos resultados, tais como uma amostra pequena e avaliação da $\mathrm{QV}$ em forma de entrevista, contudo, nossos dados estão consoantes com a literatura, que demonstram que idosos com IU referem prejuízo na $\mathrm{QV}^{24}$.

Contudo, acreditamos que a amostra estudada é representativa da população do município, por ser aleatória, apesar de estratificada e ter uma sistematização na definição dos domicílios estudados em cada região censitária. Da população selecionada inicialmente, aproximadamente $40 \%$ compareceu para a avaliação. No entanto, acreditamos que esta perda foi aleatória, exceto que houve uma pequena diferença da faixa etária, com menor comparecimento dos indivíduos mais velhos e daqueles sem renda própria.

As demais variáveis demográficas têm distribuições

TABELA 2. Gênero, idade, avaliação global da qualidade de vida e satisfação com a saúde dos idosos segundo Index de Barthel e questão única. Porto Alegre, 2006.

\begin{tabular}{|c|c|c|c|c|}
\hline & \multicolumn{2}{|c|}{ Index de Barthel } & \multicolumn{2}{|c|}{ Questão única: perde urina? } \\
\hline & Continência & Incontinência & Não & Sim \\
\hline \multicolumn{5}{|l|}{ Gênero* } \\
\hline Masculino & $122(89,7 \%)$ & $14(10,3 \%)$ & $52(71,2 \%)$ & $21(28,8)$ \\
\hline Feminino & $242(70,6 \%)$ & $101(29,4 \%)$ & $106(43,6 \%)$ & $137(56,4 \%)$ \\
\hline Idade $^{\S}$ & $69,96( \pm 7,75)$ & $71,31( \pm 7,12)$ & $69,01( \pm 7,21)$ & $70,76( \pm 7,49)$ \\
\hline Avaliação global QV & 71,96 & 64,78 & 72,86 & 67,74 \\
\hline Satisfação geral com saúde & 66,11 & 55,21 & 67,26 & 59,03 \\
\hline
\end{tabular}

*n(\%); § média (desvio padrão)

Fonte: Banco de dados do EMIPOA. 
semelhantes entre os idosos que compareceram e os que não compareceram para avaliação. A frequência das diversas condições de saúde questionadas no inquérito populacional também foi semelhante no grupo avaliado.

A proporção de mulheres e homens que referiram apresentar IU nesse estudo é semelhante aos encontrados em outros estudos ${ }^{25-28}$. Estudos epidemiológicos descrevem uma prevalência de 27,6\% de IU em mulheres entre 40 e 60 anos e de $10,5 \%$ em homens $^{29}$. A proporção de mulheres incontinentes é maior em praticamente todos os estudos. Dentre mulheres australianas houve prevalência de 36 e 35\% de perda de urina de 45 a 50 anos e 70 a 75 anos, respectivamente $^{25}$. Outros autores têm descrito prevalências menores, $26 \%$ entre mulheres japonesas $^{26}$ e $16 \%$ em mulheres dinamarquesas com idade entre 40 e 60 anos $^{27}$. Diferenças étnicas e metodológicas podem ser responsáveis pela menor prevalência descrita nesses estudos. Dados mais altos de prevalência foram observados em um estudo americano, multicêntrico, de base populacional, em que das 2.763 mulheres participantes, mais da metade referiram incontinência urinária e a média etária dessas mulheres era de 67 anos $^{28}$.

A diferença na resposta dada pelos idosos nos dois instrumentos pode ser atribuída a linguagem não compreendida, no Index de Barthel, sendo melhor entendida pelos idosos por meio da pergunta direta "perde urina". Tal observação pode, portanto, orientar para uma correta avaliação dos idosos.

Frequentemente encontrada no idoso, a IU é decorrente da senilidade dos tecidos, mas doenças contribuem para o agravamento da incontinência. Como é um estado anormal, a abordagem adequada é, na maioria dos casos, resolvida ou diminuída, porém, alterações da motivação, da destreza manual, da mobilidade, da lucidez podem ser consideradas fatores de risco para o desenvolvimento da incontinência urinária $^{6}$.

A IU causa grande impacto na qualidade de vida dos pacientes, o envolvimento do próprio paciente no seu tratamento e a necessidade de uma avaliação mais ampla, analisando como e quanto determinada doença ou intervenção do profissional de saúde atinge a qualidade de vida, são fundamentais em qualquer serviço de saúde. Diante de alterações sociais, pessoais e emocionais dos pacientes, é importante avaliar a qualidade de vida valorizando assim a opinião do paciente sobre sua própria condição de saúde ${ }^{30}$.

A Organização Mundial da Saúde e a Sociedade Internacional de Continência recomendam que pacientes com IU realizem avaliação da $Q V$, sempre levando em consideração o planejamento, o investimento e as decisões de terapêuticas, com abordagens interdisciplinares. Esta mensuração é utilizada como um dos parâmetros para avaliar não somente o tratamento utilizado, bem como serve de comparação com outros estudos ${ }^{31,32}$.

A incontinência urinária interfere diretamente nas atividades diárias dos idosos, de tal maneira que aquelas que sofrem com essa situação apresentam índices mais baixos de qualidade de vida ${ }^{25}$.

Indivíduos que apresentam perda de urina desenvolvem modificações comportamentais para se adaptar à inconveniência e reduzir os impactos dos sintomas, tais como: aumento da frequência urinária, descoberta da localização de banheiros, dietas restritivas, limitações das atividades físicas, sociais e econômicas, pois exige cuidados que implicarão em altos custos para o paciente, familiares e órgãos públicos, interferindo diretamente na qualidade de vida destes indivíduos ${ }^{30,33}$.

Forma-se um ciclo vicioso de ansiedade e sofrimento relacionado à possível perda urinária. No mesmo sentido, o sentimento de vergonha e gradativa piora da urgência miccional, que leva a um importante incômodo psicológico e variado grau de isolamento social ${ }^{30}$.

$\mathrm{Na}$ avaliação global da saúde observou-se que os idosos estudados continentes sentem-se mais satisfeitos. É importante ressaltar que o impacto destes sintomas na vida de cada indivíduo está intimamente ligado à percepção individual que cada um tem frente à severidade, tipo e quantidade da perda urinária. Além disso, esta avaliação está relacionada ao contexto cultural de cada indivíduo, apesar de em nosso estudo não verificarmos esta correlação ${ }^{34,35}$.

Pelo grande impacto da incontinência na vida das 
pacientes, os profissionais de saúde devem estar atentos em melhorar a auto-estima dos idosos e comprometidos em avaliar a QV na sua prática, auxiliando a direcionar e justificar quais intervenções necessárias que deverão ser realizadas em consultas individuais e principalmente pelo sistema de saúde.

A prevalência de IU na amostra de idosos de Porto Alegre é semelhante aos estudos encontrados em outros locais com a mesma população. Observamos algumas divergências relacionadas a QV em um estudo, provavelmente este fato se deve a quantidade amostral, bem como ao método de coleta de dados. Entretanto, na maioria dos estudos que avaliaram a $\mathrm{QV}$, a IU interfere na vida desses idosos, corroborando com nosso estudo.

Podemos verificar que a IU não deve ser considerada como uma patologia do envelhecimento, mas entendida como uma consequência de várias alterações decorrentes do processo de envelhecimento. Com o aumento da expectativa de vida é preciso qualificação e desenvolvimento de aptidões para identificar e tratar a IU na população idosa, o que pode influenciar significativamente na sua qualidade de vida.

\section{REFERENCIAS}

1. Papaléo Netto M, Ponte JR. Envelhecimento: desafio da transição do século. In: Papaléo Netto M. Gerontologia: a velhice e o envelhecimento em visão globalizada. São Paulo: Atheneu; 2005. p. 3-12.

2. Rodrigues RAP, Mendes MMR. Incontinência urinária em idosos: proposta para a conduta da enfermeira. Rev. Latino-am. Enfermagem 1994;2(2):5-20.

3. Diokono AC, Brown MB, Brock BM. Clinical and cystometric characteristics of continent and incontinent noninstitutionalized elderly. J Urol 1988; 140:567-71.

4. Resnick NM. Voiding dysfunction in the elderly. In: Yalla SV, McGuire EJ, Elbadawi A, Blaivas JG. Neurourology and Urodynamics: Principles and Practice. New York, MacMillam Publishing Company; 1998. p. 303.

5. Resnik NM, Elbadawi A, Yalla SV. Age and the lower urinary tract: What is normal? Neurourol Urodyn 1997; 12:322-30.

6. Reis RB, Colongna AJ, Martins ACP, Paschoalin EL, Tucci Jr S, Suaid HJ. Incontinência urinária no idoso. Acta Cir Bras 2003;18(5):47-51.

7. Lopes MHBM, Higa R. Restrições causadas pela incontinência urinária à vida da mulher. Rev Esc Enfermagem USP 2006; 40(1):34-41.

8. Papanicolaou S, Hunskaar S, Lose G, Sykes D. Assessment of bothersomeness and impact on quality of life of urinary incontinence in women in France, Germany, Spain and UK. BJU Int. 2005; 96(6):831-8.
9. Coyne KS, Zhou Z, Thompson C, Versi E. The impact on healthrelated quality life of stress, urge and mixed urinary incontinence. BJU Int. 2003; 92(7):731-5.

10. Lopes MHBM, Higa R. Restrições causadas pela incontinência urinária à vida da mulher. Rev Esc Enfermagem USP 2006;40(1):34-41.

11. Kelleher C. Quality of life and urinary incontinence. Baillieres Best Pract. Res Clin Obstet Gynecol 2000; 14(2):363-79.

12. Saleh N, Bener A, Khenyab N, Al-Mansori Z, Al-Muraikhi A. Prevalence, awareness and determinants of health care-seeking behaviour for urinary incontinence in Qatari women: a neglected problem? Maturitas 2005;50(1):5865.

13. Fultz NH, Burgio K, Diokno A, Kinchen KS, Obenchain R, Bump RC. Burden of stress urinary incontinence for community-dwelling women. Am J Obstet Gynecol 2003; 189(5):1275-82.

14. Souza ACA et al. Avaliação transdisciplinar de idosos de Porto Alegre. Porto Alegre: PUCRS [IGG]; 2005.

15. Roach $\mathrm{S}$. Introdução à enfermagem gerontológica. Rio de Janeiro: Guanabara Koogan; 2003.

16. Universidade Federal do Rio Grande do Sul (UFRGS). [acessado 2007 ago 06]; Disponível em: <http://www.ufrgs.br/psiq/breve.pdf>.

17. De Granger CV, Albrecht GL, Hamilton BB. Outcome of cromprehensive medical rehabilitation: measures of PULSES profile and the Barthel Index. Arch Phys Med Rehabil.1979;60(4):145-154.

18. Shah S, Vanclay F, Cooper B. Improving the sensitivity of the Barthel Index for stroke rehabilitation. J Clin Epidemiol. 1989;42(5):703-9.

19. Sanches MAS. A dependência e suas implicações para perda de autonomia: estudos das representações para idosos de uma unidade ambulatorial geriátrica [dissertação]. Habana (Cuba): Universidad de La Habana; 1998.

20. Cid-ruzafa J, Damián-Moreno J. Valoración de la discapacidad física: el índice de Barthel. Esp Salud Publica. 1997;71(2):127-37.

21. Mahoney $\mathrm{FI}$, Bathel DW. Functional evaluation: the Barthel Index. Maryland State Med J. 1965;14:56-61.

22. Fleck MPA, Louzada S, Xaviera M, Chachamovich E, Vieira G, Santos L, Pinzon V. Aplicação da versão em português do instrumento de avaliação da qualidade de vida "WHOQOL-bref". Rev Saúde Pública. 2000; 34(2):178-83.

23. Conselho Nacional de Saúde (BR). Resolução 196-96, de 10 de outubro de 1996. Diretrizes e normas reguladoras de pesquisa envolvendo seres humanos. Brasília (DF): Ministério da Saúde; 1996.

24. Teleman P, Lidfeldt J, Nerbrand C, Samsioe G, Mattiasson A. Lower urinary tract symptoms in middleaged women-prevalence and attitude towards mild urinary incontinence: a communitybased population study. Acta Obstet Gynecol Scand. 2005;84(11):1108-12.

25. Chiarelli $P$, Brown W, McElduff $P$. Leaking urine: prevalence and associated factors in Australian women. Neurourol Urodyn 1999;18:567-77.

26. Ushiroyama T, Ikeda A, Ueki M. Prevalence, incidence, and awareness in the treatment of menopausal urinary incontinence. Maturitas 1999; 33:127-32.

27. Moller LA, Lose G, Jorgensen T. The prevalence and bothersomeness of lower urinary tract symptoms in women 40-60 years of age. Acta Obstet Gynecol Scand 2000;79:298-305 
28. Brown JS, Grady D, Ouslander JG, Herzog AR, Varner RE, Posner SF. Prevalence of urinary incontinence and associated risk factors in postmenopausal women. Hert \& Estrogen/Progestin Replacement Study (HERS) Research Group. Obstet Gynecol 1999; 94:66-70.

29. Minassina VA, Drutz HP, Al-Badr A. Urinary incontinence as a worldwide problem. Int $\mathrm{J}$ Gynecol Obstet. 2003;82(3):327-38.

30. Davila GW, Neimark M. The overactive bladder: prevalence and effects on quality of life. Clin Obstet Gynecol. 2002; 45(1):173-81.

31. The World Health Organization Quality of Life assessment (WHOQOL): position paper from the World Health Organization. Soc Sci Med. 1995;41(10):1403-9.

32. Rodrigues RAP, Zago MMF. Incontinência urinária no idoso: assistência de enfermagem. Rev. Escola de Enfermagem USP. $1991 \mathrm{Abr} ; 25(1): 21-8$.

33. Blaivas JG, Appell RA, Fantl JA, Leach G, McGuire EJ, Resnick NM, et al. Standards of efficacy for evaluation of treatment outcomes in urinary incontinence: recommendations of the Urodynamic Society. Neurourol Urodyn. 1997;16(3):145-7.

34. Papanicolaou S, Hunskaar S, Lose G, Sykes D. Assessment of bothersomeness and impact on quality of life of urinary incontinence in women in France, Germany, Spain and UK. BJU Int. 2005;96(6):831-8.

35. Kelleher C. Quality of life and urinary incontinence. Baillieres Best Pract Res Clin Obstet Gynecol. 2000; 14(2):363-79.

\section{Endereço para correspondência:}

Karin Viegas

Av. Ipiranga, 6681. Prédio 12. $8^{\circ}$ andar.

Porto Alegre/RS - CEP 90619-900

Telefone: +55 51 3320-3646

Email: karin.viegas@pucrs.br 